\title{
HLA-DR Antigen
}

National Cancer Institute

\section{Source}

National Cancer Institute. HLA-DR Antigen. NCI Thesaurus. Code C16692.

Encoded by multiple HLA-DRA and HLA-DRB genes in a complex variable $5 \mathrm{cM}$ region of MHC between HLA-B and -D, HLA-DR Antigens are Class II histocompatibility transmembrane glycoprotein heterodimers of alpha (heavy, 35-kD) and beta (light, 27 kD) chains. Located predominantly on B cells and macrophages, HLA-DR antigens function in antigen presentation to regulatory $T$ cells in the immune response and in self/nonself discrimination. Invariant alpha and polymorphic beta chains have glycosylated N-termini, hydrophobic membrane regions, and hydrophilic C-termini. The heterodimer consists of 4 extracellular domains; invariant alpha-1, polymorphic Nterminal beta-1, and conserved Ig-like alpha-2 and beta-2. Alpha- 1 and alpha- 2 contain disulfide loops. Beta-1 contains 2 small variable regions. Alpha sequences have relatively simple structure; beta chains carry the major polymorphic determinants. 